\title{
Características de un dispositivo ambulatorio intensivo en el tratamiento de trastornos por uso de sustancias
}

\author{
Characteristics of an intensive outpatient device in the treatment of Substance use Disorders
}

\section{Diana Milena Berrio Cuartas',Ana Karina Hurtado Márquez',Timoteo Bovino'}

\begin{abstract}
Resumen
El trastorno por uso de sustancias (TUS) altera significativamente el rendimiento social, familiar y laboral del paciente, así como su salud física y mental. El Dispositivo Pavlovsky (DP), cuyas características se describen en este artículo, tiene una modalidad de tratamiento de carácter ambulatorio e intensivo para usuarios cuyo estado de gravedad no requiere internación, pero sí un abordaje de mayor intensidad que los tratamientos ambulatorios tradicionales. El DP, creado en Buenos Aires, Argentina, tiene una batería de propuestas terapéuticas protocolizadas dentro de un marco preestablecido, adaptadas a las necesidades del paciente. Debido a su carácter intensivo, brinda mayor contención psíquica y promueve la abstinencia en los usuarios, quienes por su patología presentan rasgos comunes de ambivalencia a suspender el consumo de las sustancias problema. La propuesta terapéutica se caracteriza por ofrecer y promover el uso de herramientas y estrategias para la vida cotidiana a partir de terapia grupal intensiva, individual, consulta psiquiátrica, toxicológica, asistencia a talleres, formación de una red participativa, a los cuales se les brinda un espacio grupal de contención, orientado a la psicoeducación en el acompañamiento al paciente. El DP cuenta con equipos interdisciplinarios con diversos marcos teóricos. Sus profesionales mantienen comunicación fluida, con espacios formales de intercambio, actuando en la direccionalidad de los tratamientos en general y profundizando cada caso en particular.
\end{abstract}

Palabras clave: Dispositivos ambulatorios - Abuso de sustancias - Conducta adictiva, Tratamiento intensivo ambulatorio - Intervenciones grupales.

\section{Abstract}

The Substance Use Disorder (SUD) alters the patient's social, family and working performance significantly, together with Mental and Physical Health. The Dispositivo Pavlovsky (DP) is an outpatient and intensive treatment modality for users/ clients that do not require an inpatient facility but do need a more intense approach than traditional/classic out patient treatment. The DP has a variety of therapeutic of fers with a protocol in a pre established setting, adapted to the patient's needs. The treatment intensive given characteristic, it provides a better psychical support and promotes abstinence among the users/clients, who, because of their pathology, present common features of ambivalence towards suspending the consumption of the problematic substance. Overall, the therapeutic proposal is characterized by offering and promoting the use of tools and strategies for the daily life, such as intensive Group Therapy Setting, Individual Therapy, Psychiatric Consultation, Toxicology consultation, attendance to workshops, creating a participatory network, that provides a group setting support oriented to wards psychoeducation, and accompaniment of the patience. The DP has interdisciplinary teams with diverse theoretical frameworks. These professional skeep a fluid communication, with formal exchange settings, intervening in the directionality of the treatment in general and delving deep in each particular case.

Keywords: Outpatient treatment - Substance abuse - Addictive behavior - Intensive outpatient treatment - Group intervention.

RECIBIDO 30/8/202I - ACEPTADO 6/I2/202I

1.Médico Psiquiatra. Miembro del equipo asistencial del Dispositivo Pavlovsky.

Autor de referencia:

Diana Milena Berrio Cuartas

dianaoasis@yahoo.com

Lugar de realización del trabajo: Centro asistencial del Dispositivo Pavlovsky. 


\section{Introducción}

Según la Organización Panamericana de la Salud (OPS) " 4,4 millones de hombres y 1,2 millones de mujeres de América Latina y el Caribe sufren trastornos causados por el uso de drogas en algún momento de su vida" (Organización Panamericana de la Salud, 2021). En el Estudio Epidemiológico de Salud Mental en población general de la República Argentina publicado en el 2018 el 10.4\% de los encuestados presentaban algún tipo de trastorno por consumo de sustancias (Stagnaro et al, 2018).

El trastorno por uso de sustancias (del inglés TUS) está definido como una entidad clínica crónica con alteración neurobiológica. Confluyen en la etiología de este trastorno factores genéticos, psicosociales y ambientales, que se caracterizan por conductas como el craving, problemas en el control del uso de la sustancia, uso compulsivo de la misma y continuidad del consumo, a pesar del daño físico y/o psíquico (Calvo-García et al., 2016; Portero Lazcano, 2015).

La obtención y el consumo de la sustancia alteran significativamente el rendimiento social, familiar y laboral del paciente. El TUS es muy complejo y también lo es su abordaje. Este trastorno aparece en cualquier sujeto inmerso en un escenario cultural específico, en búsqueda de novedad, gratificación inmediata a la búsqueda de estímulos y alivio de las tensiones rutinarias o del malestar psíquico (Capece, 2008). Los resultados terapéuticos dependen en gran medida de la adherencia al tratamiento (Velázquez Benítez et al., 2016).

A finales de los años ochenta y principios de los noventa del siglo XX, apoyados por la investigación neurológica y los hallazgos de los fundamentos neurológicos a la adicción (el circuito de recompensa cerebral), los enfoques cognitivos comenzaron a ser los más usados para el abordaje de las adicciones (Capece, 2008).

Actualmente, en el tratamiento de las conductas adictivas, se destaca la aplicación de desarrollos teóricos como el de Prochaska y Di Clemente con la "entrevista motivacional" (Templos Nuñez, \& Navarrete, 2014), las estrategias de "prevención de recaídas" de Marlatt y Gordon (Risso et al., 2019), el "manejo de contingencias” de Silverman (García Rodríguez, 2008), las aproximaciones motivacionales de Miller (Templos Nuñez, \& Navarrete, 2014), la psicoeducación y el modelo de reducción de daños (Velázquez Benítez et al., 2016).

Los pacientes afectados por el TUS circulan por diferentes dispositivos de tratamiento en función de su gravedad clínica. Si ésta implica un riesgo vital in- minente, como en el caso de las urgencias clínicas, se indica la internación hospitalaria como primera medida para el cuidado general y evaluación de estrategias terapéuticas a fin de atender el síndrome de abstinencia (Echeburúa et al., 2004; López-Núñez et al., 2016). Estos cuidados suelen llevarse a cabo en servicios de toxicología o de clínica médica, seguidos, dependiendo del caso, de una internación en servicios de salud mental. Luego de la compensación del cuadro agudo se suelen indicar diversos dispositivos para el tratamiento ulterior, como comunidades terapéuticas, grupos terapéuticos o abordajes tradicionales de salud mental de tipo ambulatorios. Estos espacios, generalmente desarticulados entre sí, tienen poca eficacia. A medida que se ha avanzado en la investigación del TUS se ha reconocido la importancia de los tratamientos integrales que involucran el entorno social y la comunicación o conocimiento de las diferentes instituciones que, eventualmente, intervienen en la problemática a tratar.

El National Institute on Drug Abuse de los EE. UU., ha publicado los "Principios de tratamientos para la drogadicción: Una guía basada en las investigaciones. Principios de tratamientos eficaces" (NIDA, 2020), los cuales de manera resumida definen que un tratamiento eficaz debe considerar:

- El TUS como una enfermedad compleja pero tratable.

- El tratamiento para el TUS debe estar disponible de manera permanente.

- El tratamiento eficaz para el abandono del consumo de sustancia debe valorar las diversas necesidades de la persona: alteraciones clínicas (HIV y hepatitis, enfermedades metabólicas como diabetes, hipercolesterolemia o alteraciones cardiacas), psicológicas, sociales, y legales.

- Un tratamiento eficaz requiere su mantenimiento por un periodo de tiempo adecuado.

- La eficacia del tratamiento se basa en la implementación de grupos y actividades que ayuden a desarrollar habilidades para sustituir el consumo de la sustancia y actividades vitales constructivas y gratificantes.

- Se debe considerar tratamientos con psicofármacos para el manejo de síntomas de abstinencia y sustitución de la sustancia como es el caso de los opioides.

- Evaluar permanentemente el tratamiento de cada paciente y modificarlo en caso de que sea necesario. 
- La desintoxicación médica es sólo la primera etapa del tratamiento para la adicción y por sí sola rara vez es suficiente para ayudar a los pacientes a lograr abstinencia y recuperación.

- El tratamiento no tiene que ser voluntario para ser eficaz.

- Pueden ocurrir recaídas durante el tratamiento por consumo de sustancias.

En el presente artículo, se describe el esquema de tratamiento en el Dispositivo Pavlovsky (DP), un Dispositivo Ambulatorio Intensivo (DPI) para pacientes con TUS, cuya sede principal se encuentra en la Ciudad de Buenos Aires, Argentina.

\section{Esquema de tratamiento en el DAl del DP y descripción de las herramientas para pacientes}

El DP despliega una estrategia a cargo de un equipo de profesionales ante un problema de salud mental grave ocasionado por el TUS, considera la rehabilitación y las necesidades de apoyo social específicas a la persona afectada, durante un período de tiempo indefinido que reduce la necesidad de internación y aumenta la adherencia al tratamiento. Este DAI es importante para el tratamiento de patologías crónicas (Dieterich et al., 2017). La definición de la APA (American Psychiatric Association, 1995) establece que un tratamiento es intensivo, cuando el usuario asiste al mismo un mínimo de tres horas a razón de tres veces por semana. Una alta frecuencia de contacto entre el equipo y el paciente es una variable documentada de mayor eficacia del programa (American Psychiatric Association, 1995; Timko, 2008).

El tratamiento intensivo se divide en tres etapas: admisión, tratamiento y mantenimiento. La puerta de entrada al dispositivo es la entrevista de admisión. A partir la página Web de la institución se ofrece respuesta inmediata a cualquier consultante. La entrevista inicial puede ser realizada por distintos miembros del equipo. El 50\% de los consultantes concurren a la admisión y de éstos el 50\% comienza la experiencia terapéutica (Pavlovsky et al., 2020). La respuesta debe ser pronta y eficaz.

Se practica una primera evaluación del consultante y de las circunstancias vinculadas al consumo: ¿Cuáles sustancias consume?, ¿desde cuándo consume sustancias?, ¿en qué contexto consume? La respuesta a esas preguntas brinda una primera impresión de las implicaciones personales, familiares y sociales del consultante. En la admisión al DAI se evalúa si éste es la mejor alternativa para el paciente, o si se requieren otras instancias previas: internación para desintoxicación o tratamiento de enfermedades clínicas agudas. Se indaga acerca de los motivos del inicio del tratamiento y se inicia la etapa de pre-contemplación o contemplación del abandono del consumo (Templos Nuñez, \& Navarrete, 2014).

Es importante mencionar que, aunque el DAI está indicado para patologías severas e incluso para pacientes recién externados de tratamientos de internación por salud mental, si el paciente no está compensado psiquiátricamente, no cuenta con red de apoyo social o no tiene intenciones de seguir alguna de las indicaciones iniciales de los profesionales, no puede ser admitido, al menos en ese momento. Se tienen en consideración "los criterios de exclusión de Fishman" planteados en 1999: "psicosis aguda, psicosis crónica con deterioro neuropsicológico severo, retraso mental y comportamiento o ideación que sugiere un riesgo para sí mismo o para otros" (Fishman et al., 1999).

En el tratamiento del trastorno por consumo de alcohol se han descrito técnicas para tratar los procesos automáticos asociados al consumo. Se está investigando un conjunto de programas de capacitación computarizados, asociados al entrenamiento de modificación de sesgo cognitivo (CBM) los cuales buscan cambiar el sesgo de atención, de enfoque y de memoria (Bratti-van der Werf et al., 2018). En el caso del DP las herramientas están elaboradas según el modelo Patient Centered Care (PCC), es decir, "centradas en el paciente", que están relacionadas con el cuidado integral, la psicoterapia individual, el compartir toma de decisiones y crear vínculo terapéutico de acuerdo a las características personales del paciente (Marchand et al., 2019).

En el DP se aplica la app VLOV. La iniciativa está orientada a que el paciente se vincule activamente durante y con su tratamiento. El mismo ingresa su información personal, el nombre de su terapeuta individual y los datos de su obra social para ser usados en caso de emergencia. Así, el paciente tiene todos los días un conteo del tiempo que lleva de abstinencia (dividido en horas, días y meses) que tendrá que reiniciar en caso de recaída en el consumo. Con la VLOV el paciente registra cómo está su estado de ánimo ("En riesgo de recaer", "Excelente", "Muy bien", "Regular", "No sé cómo me siento"). Esto sirve de monitoreo del estado de ánimo para el paciente mismo y por supuesto, para sus terapeutas, quienes pueden decidir intervenciones farmacológicas, frecuencia de grupos 
terapéuticos $\mathrm{u}$ otras indicaciones que eviten recaídas en el consumo. Además, la VLOV cuenta con tracks de meditación que pueden ayudar a disminuir la ansiedad, el deseo de consumo, etc. El paciente también cuenta con un botón directo al teléfono de emergencia que señaló a su ingreso, en caso de tener que activar el protocolo de recaída en el consumo y otro botón denominado "Necesito ayuda", que da las opciones de "Llamar a alguien cercano", "Llamar a mi terapeuta", "Llamar a emergencias", "Meditar" y "Repasar protocolo". Todos los días el paciente recibe un e-mail en su casilla personal, recordando la agenda de grupos terapéuticos y talleres. Esto mejora la adherencia e implicación en el tratamiento (Figura 1).

La etapa de admisión incluye los pacientes que recién son ingresados al dispositivo. En ella es fundamental cumplir con varios requisitos, entre ellos: evaluación por la toxicóloga del equipo quien reali-

Figura I. Aplicación VLOV
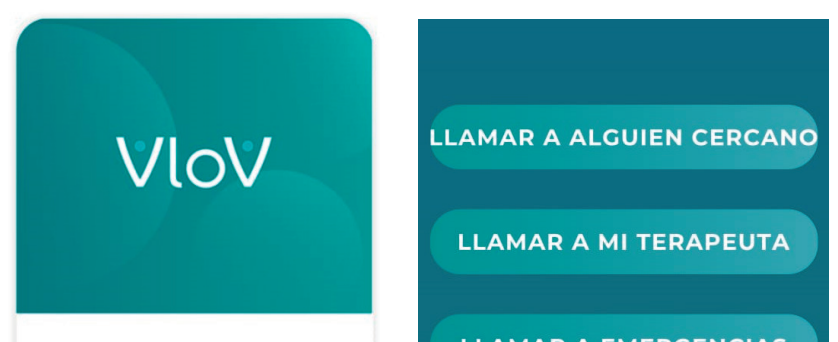

LLAMAR A EMERGENCIAS

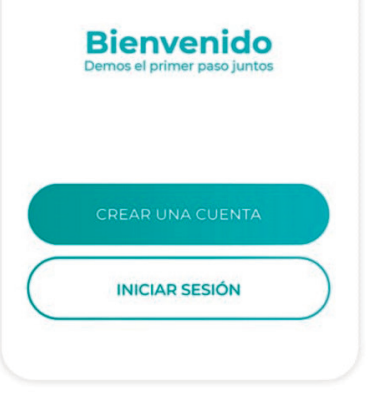

za chequeo clínico completo, antecedentes clínicos y estado clínico asociado a la sustancia problemática. Además, se incluye un chequeo odontológico y nutricional. Se realiza una evaluación psiquiátrica inicial y la designación de un psicoterapeuta individual con quien el paciente comienza un seguimiento semanal, aunque, en algunos casos, la frecuencia es mayor (ver Cuadro 1).

En esta etapa inicial del tratamiento se define la red de apoyo con la que cuenta el paciente, entre las características de la red de apoyo se espera que cada paciente tenga al menos una persona como red; ésta funciona como sistema de aviso y alerta de dificultades y problemas y potencia y amplifica los principios terapéuticos del programa en el ámbito cotidiano ( $\mathrm{Pa}-$ vlovsky et al., 2020).

Los pacientes de admisión cuentan con dos grupos diarios a los que se agrega una "clase" de "entrenamiento en herramientas", de frecuencia semanal. También pueden incluirse en talleres. Las primeras cuatro semanas representan un momento sensible por la alta posibilidad de abandonos documentados en la bibliografía, en particular para aquellos con una dependencia a la cocaína (Veach et al., 2000; Barnicot, \& Crawford, 2019).

El paciente que cumple con los objetivos pautados para la primera etapa, pasa a tratamiento. En esta etapa los pacientes ya tienen entrenamiento en las herramientas disponibles, como el protocolo de crisis (llamada a su servicio de asistencia clínica urgente en caso de recaída en el consumo para la evaluación clínica en el lugar) y la VLOV; comienzan su asistencia a grupos terapéuticos diarios y establecen un vínculo terapéutico con su psicólogo y su psiquiatra. Uno grupos terapéuticos es específico para "prevención de recaídas". Una vez a la semana se realizan "encuentros de red" que implican la asistencia de los miembros de las redes de apoyo social con las que cuentan los

Cuadro I. Objetivos de la entrevista de admisión

\begin{tabular}{c|c|c|c}
\hline $\begin{array}{c}\text { I- Promover un encuentro } \\
\text { empático }\end{array}$ & $\begin{array}{c}\text { 2- Identificar contactos } \\
\text { significativos }\end{array}$ & $\begin{array}{c}\text { 3- Detectar trastornos } \\
\text { mentales comórbidos }\end{array}$ & 4- Evaluar severidad \\
\hline 5- Evaluar riesgo de vida & 6- Determinar diagnóstico & $\begin{array}{c}\text { 7-Determinar capacidad } \\
\text { funcional }\end{array}$ & $\begin{array}{c}\text { 8- Identificar recursos de } \\
\text { funcionamiento general }\end{array}$ \\
\hline $\begin{array}{c}\text { 9- Identificar variables } \\
\text { socioculturales relevantes } \\
\text { para el tratamiento }\end{array}$ & $\begin{array}{c}\text { I0- Identificar cuál es } \\
\text { el problema dominante }\end{array}$ & $\begin{array}{c}\text { II- Determinar el estadio } \\
\text { del proceso de cambio }\end{array}$ & $\begin{array}{c}\text { I2- Configurar un plan } \\
\text { integral de tratamiento }\end{array}$ \\
\hline
\end{tabular}


pacientes, con el objetivo de entrenar también a los familiares y amigos en el proceso de recuperación del paciente y en el apoyo que requiere cada uno para el mantenimiento de la abstinencia y la recuperación. La red de apoyo también es entrenada para actuar en caso de consumo y en la activación del protocolo de crisis.

Las indicaciones de asistencia las define el equipo tratante. En caso de que el paciente no cumpla adecuadamente con estas indicaciones, es llamado por el coordinador del DAI para consultar las razones del incumplimiento e incentivar la participación activa en los grupos terapéuticos y talleres correspondientes.

El DAI también cuenta con otros espacios terapéuticos como los talleres de actividad física, espacio creativo, cine debate, meditación. También se encuentran en agenda "grupos específicos del dispositivo" como el taller para mujeres en tratamiento, el de integración sociolaboral, el de pares, el de cesación tabáquica y el de terapia dialéctico conductual (DBT). Éste último ha demostrado ser eficaz en el tratamiento del trastorno límite de la personalidad y se está implementando para el tratamiento por consumo de sustancias, especialmente para entrenar a los pacientes en la identificación emocional y su control (Sánchez et al., 2011).

El DP cuenta con el "Dispositivo de Noche", destinado a pacientes en estadio pre-contemplativo frente al cambio, que tienen una severidad similar a los demás pacientes admitidos, pero expresan una negativa a participar en un programa de alta exigencia, cómo lo supone el programa general. Actualmente se está estudiando la adherencia al tratamiento de los pacientes que participan y los factores del Índice de Severidad de Adicción (Urshel et al., 2003) diferenciales entre ambas modalidades.

La tercera etapa del tratamiento corresponde al "mantenimiento". En ella los pacientes están adheridos a la propuesta terapéutica y cuentan con un espacio específico para continuar. El tiempo estimado de permanencia del paciente en la propuesta asistencial oscila entre los 12 a 18 meses con abstinencia global. Esto se asocia al modelo MATRIX (González et al., 2013), según el cual la permanencia del paciente en tratamiento es un predictor a futuro de una evolución más favorable. La abstinencia global disminuye el riesgo de recaídas en el consumo (Veach et al., 2000; Pavlovsky et al., 2020).

En el DP se ha observado una relación directamente proporcional entre la frecuencia semanal y una evolución positiva en términos de adherencia y problematización del consumo. En algunos casos puntuales, se indica la "Asistencia Diaria" (AD) a razón de siete días por semana.

A todo lo largo del tratamiento en DP se completa una Historia Clínica (HC) de tipo electrónica.

En el Cuadro 2 se brinda un esquema de las etapas del tratamiento que se han enumerado.

Cuadro 2. Etapas del tratamiento en el dispositivo ambulatorio intensivo (DAI)

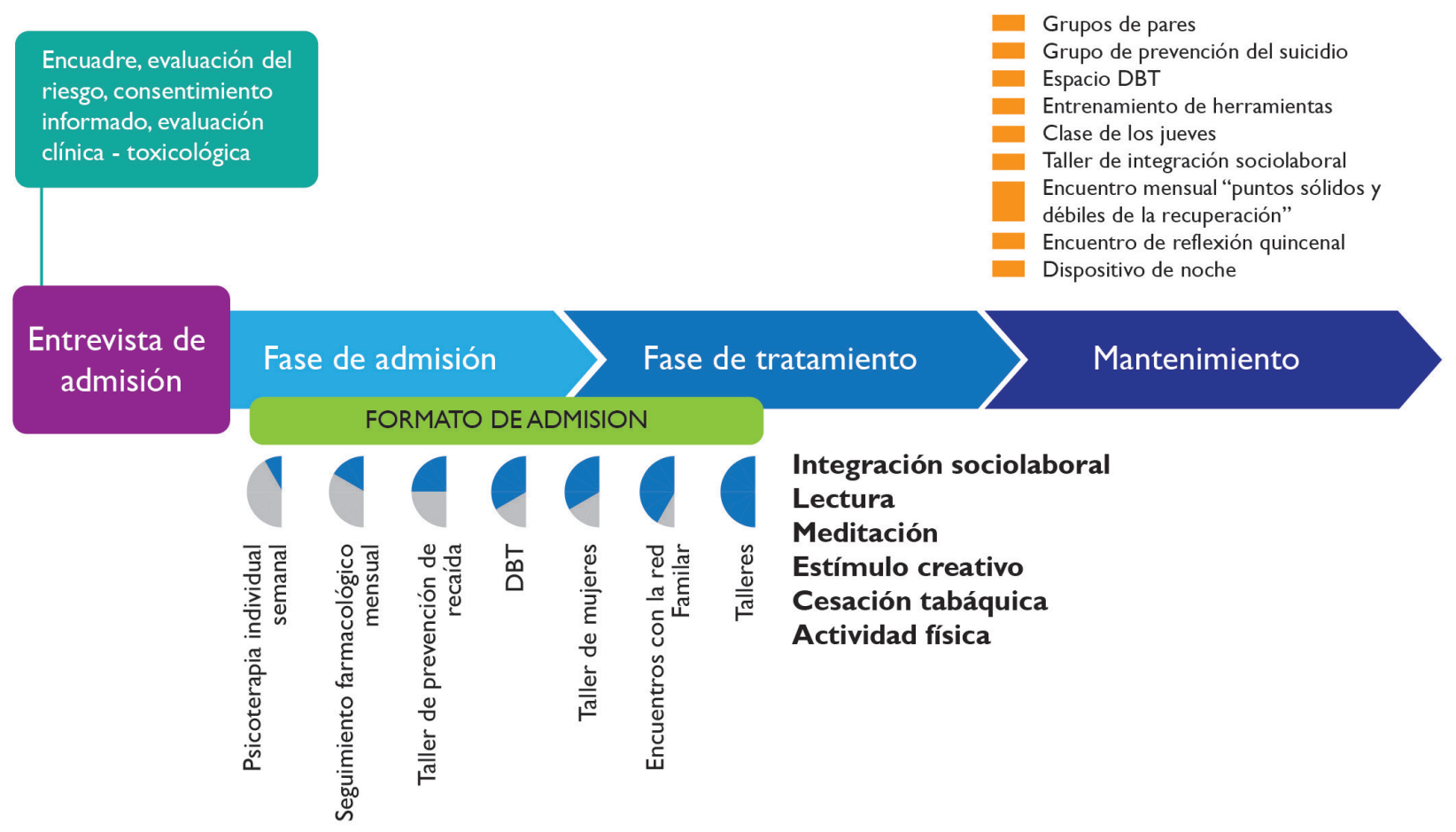




\section{Características del equipo terapéutico}

Enrique Pichón-Rivière describe que “... a mayor heterogeneidad de los miembros de un grupo, heterogeneidad regida a través de la diferenciación de los roles desde los cuales cada miembro aporta su bagaje de experiencias vividas, sus conocimientos adquiridos, como así también sus diferencias, logra la suma de información que se potencia dando lugar a un cambio cualitativo y a una mayor productividad" (Jasiner, 2008). Esta es la base fundamental del intercambio entre profesionales del DP.

La necesidad de la organización de las tareas genera una dinámica de cambio (Del Cueto, \& Fernández, 2002), lo que establece el valor simbólico de la reunión de equipo. Se busca que la reflexión consciente ofrezca un consenso en decenas de decisiones que impactan en la constitución de calidad de vida de los pacientes graves que presentan una dependencia a algún tipo de sustancias y de su entorno familiar (Nutt et al., 2007). Se realiza un análisis detallado de las situaciones emergentes, sin que estas superen la voracidad de la urgencia. El DP funciona como una "maquinaria en constante movimiento" (Pavlovsky et al., 2020).

El trabajo en equipo se orienta a cumplir distintas funciones que apuntan a la diversidad de formaciones disciplinarias y construye un conocimiento a partir de lo grupal, por lo que establece la necesidad de generar un vínculo colaborativo, de confianza y motivación que invita a los pacientes a participar de los diferentes espacios individuales y grupales como talleres, y la adquisición en la práctica de habilidades como es el protocolo de crisis, instaurando un abordaje integral que pueda ofrecer un tratamiento acorde a las necesidades del consultante.

Vale aquí señalar el concepto de apuntalamiento de Kaës (1996). Éste incluye la idea que el apoyo es equivalente a sostén, la relación de apoyatura es recíproca y simultánea entre quienes apoyan y quien se siente apoyado, como, por ejemplo, los miembros del equipo asistencial del dispositivo se sostienen entre sí. Entregan su narcisismo individual al grupal permitiendo crear dinámicas que favorezcan la resolución de situaciones de crisis social y personal de los pacientes en consumo.

En el marco del tratamiento, el vínculo de confianza sólido que se construye en el día a día permite que algunos pacientes comiencen a problematizar su consumo, se preparen para cambiar y eventualmente lo hagan (Pavlovsky et al., 2020). Es común que en este tipo de pacientes lo repetido hasta el hartazgo sea que todos ellos mienten y que sólo algunos de ellos quieren mejorar, ubicándolo en un rol devaluado y poco confiable. El equipo del DP pretende invertir esa ecuación y poner el acento en convertirse en un recurso confiable para el consultante.

La confianza hacia el equipo se construye en base a una serie de premisas: honestidad con el paciente, condiciones de contacto con la red claras y transparentes, disponibilidad cotidiana, coherencia entre aquello que señalamos como preocupación y nuestro accionar, presencia en las crisis, compromiso ético y clínico de evaluar periódicamente si el tratamiento funciona o no, formulación de objetivos verificables, solicitud de una segunda opinión cuando es necesario, intervención en caso de riesgo cierto e inminente para proteger la seguridad y la vida del paciente y/o la de sus allegados. Por último, las intervenciones siguiendo el estilo y la filosofía de un DAI, son realizadas activamente a partir de una escucha receptiva y reflexiva, sin juzgar, que permiten validar los sentimientos que vivencian los pacientes y sus familias en el proceso terapéutico.

\section{La evaluación médica psiquiátrica en el DAI}

El médico psiquiatra tiene como responsabilidad una serie de funciones específicas: identificar el riesgo y actuar en consecuencia, evaluar la comorbilidad psiquiátrica -si es necesario implementar una estrategia psicofarmacológica para aliviar síntomas de abstinencia y/o reducir el craving, así como tratar un trastorno psiquiátrico asociado-; derivar a niveles de mayor complejidad médica (ej: síndromes de abstinencia severos) o a efectores de la red de salud mental (ej: riesgo cierto e inminente); promover las interconsultas con colegas médicos, monitorear en términos psicopatológicos la evolución del paciente; informar los efectos del abuso de sustancias sobre su salud, discutir opciones de abordaje a su problemática de consumo; reevaluar periódicamente si el dispositivo actual de tratamiento aplicado es el adecuado, comparar impresiones subjetivas con el terapeuta y entrevistar a la familia o red del paciente, como funciones principales.

Cada paciente es asistido por un psiquiatra, con independencia de si tiene indicación farmacológica o no. A tal efecto se utiliza el modelo de trabajo para la entrevista psiquiátrica del Manual de trabajo del DP para el tratamiento de consumos problemáticos $(\mathrm{Pa}-$ vlovsky et al., 2020).

El psiquiatra suele realizar una agenda de temas sobre el estilo de consumo en forma colaborativa con 
el paciente. Es frecuente que el paciente desee interrumpir una sustancia-problema, pero no abandonar todo tipo de consumo. Lejos de ser un tema de confrontación, se trabaja ese motivo de consulta; no sin señalar que la propuesta de tratamiento propone una abstinencia global de sustancias psicoactivas, en razón de que mantener el consumo de sustancias se asocia a una peor evolución. Los pacientes suelen ser precisos en distinguir cualitativamente qué sustancias representan un problema mayor para ellos y cuáles no; aunque suelen fallar notablemente en dimensionar el impacto del consumo (de cualquier sustancia) en el desencadenamiento de una recaída.

En las instancias iniciales de tratamiento se realiza un screening cuyos resultados aportan datos complementarios para evaluar la dependencia de la sustancia o el grado de abstinencia. A tal efecto se emplean las siguientes herramientas:

- Drug Screening Inventory (Tarter, \& Hegedus, 1991): tiene por objeto identificar áreas problemáticas de la vida de relación, evaluar el consumo de drogas en adolescentes y adultos y permite categorizar y cuantificar los indicadores del problema en su relación con diferentes trastornos de ajuste psicosocial.

- CAGE (1968) (Campo-Arias et al., 2009): es un cuestionario auto-administrado breve y sencillo, con gran utilidad en atención primaria para el screening de alcoholismo.

- Test AUDIT (1987) (Babor et al., 2001): elaborado por la Organización Mundial de la Salud (OMS), es un cuestionario breve y auto-administrado que explora cantidad y frecuencia del consumo, presencia de trastornos relacionados con el alcohol, sintomatología de dependencia y consecuencias negativas del consumo.

- Test de Fagerström (1978) (Arias Gallegos et al., 2018): es un test breve, fácil de realizar, tipo cuestionario de respuesta múltiple que evalúa diferentes aspectos del comportamiento del fumador $y$ estima el nivel de adicción.

- Evaluación Neurocognitiva: es imprescindible por múltiples causas: 1) el abuso a largo plazo se manifiesta con deterioro del control ejecutivo (inhibitorio) de la memoria de trabajo y de la toma de decisiones, 2) los déficit cognitivos influyen en el nivel funcional del paciente en su cotidianidad, 3 ) puede ser un factor de vulnerabilidad para el inicio y el mantenimiento de una adicción, 4) puede facilitar el desarrollo de recaídas, 5) puede limitar los logros del paciente en un tratamiento. Los procesos cognitivos más afectados son la atención, la memoria y las funciones ejecutivas, y es de buena práctica que exista una información neuropsicológica en la evaluación inicial (Bagnati, 2017). La herramienta que utilizamos al ingreso es el Test de evaluación cognitiva de Montreal (Montreal Cognitive Assessment, MOCA) (Loureiro et al., 2018). El MOCA es una herramienta de cribado, de uso libre, desarrollada originalmente en 2005, precisamente como respuesta a la dificultad para identificar la presencia de deterioro cognitivo con el MMSE. Constituye una batería de pruebas breves, de fácil administración, con una duración aproximada de 10 minutos, cuyo propósito es detectar la presencia de deterioro cognitivo leve y diferenciarlo de alteraciones cognitivas consecuentes al envejecimiento normal.

En términos de diagnóstico psiquiátrico con objetivos técnicos e instrumentales, en el DP se utilizan los criterios incluidos en los manuales diagnósticos vigentes (CIE 11 y DSM 5).

Otra herramienta empleada son los métodos de screening de detección de drogas en cualquier momento del tratamiento. Desde el momento en que ingresan al dispositivo los pacientes saben de la utilización de esta herramienta que sirve para que, a partir de un eventual resultado positivo, tomen dimensión de su consumo y se pueda trabajar sobre ello. Si el paciente se niega a realizar esas mediciones se considera el resultado como positivo. Lejos de tomar medidas punitivas o asumir un rol "policíaco", el equipo interviene de diferentes maneras para fomentar la abstinencia global y la adherencia al tratamiento. Los indicadores de un tratamiento exitoso se enumeran en el Cuadro 3.

\section{La intervención psicofarmacológica}

La intervención psicofarmacológica en los casos de adicciones suele obedecer a dos maneras de prescripción igualmente equivocadas: el uso excesivo e indiscriminado de fármacos, o, por el contrario, su no utilización, aunque se sabe que su eficacia está probada. El psiquiatra debe tener presente que la estrategia psicofarmacológica es una de las variables que puede fortalecer, mediante un uso racional, las probabilidades de que el paciente interrumpa su consumo de sustancias. Los fármacos utilizados para sedar al paciente con el objetivo de "que no consuma" o los estabilizantes del estado de ánimo "para disminuir los impulsos 
Cuadro 3. Indicadores de un tratamiento exitoso dispositivo Pavlovsky

I. Acceso al sistema médico para el cuidado de problemas clínicos

2. Conformar una red de trabajo con el paciente

3. Disminuir el consumo de sustancias: frecuencia, cantidad, repercuciones

4. Interrumpir conductas de riesgo para sí y para terceros

5. Estabilizar problemas psiquiátricos

6. Incluir al paciente en un tratamiento de salud mental

7. Activar el proceso de cambio

8. Estimular la participación activa

9. Desarrollar una relacion de confianza con el equipo tratante y compañeros de grupo

10. Incorporar gradualmente herramientas del proceso de recuperación

a consumir", son de poca utilidad. En la actualidad, faltan estudios que aporten evidencia respecto a cómo complementar los abordajes psicoterapéuticos con los abordajes farmacológicos. Es así que predominan esquemas de polifarmacia sin evidencia científica, que, en muchos casos, sólo empeoran la evolución del paciente. En relación con la medicación se produce una situación sorprendente: una subutilización notable de medicamentos aprobados para dependencias específicas (nicotina, alcohol, opiáceos) y una sobre utilización de fármacos para casos en donde aún no se pueden extraer conclusiones firmes acerca del beneficio (cannabis, cocaína).

La medicación es útil y necesaria en el tratamiento sintomático de los síntomas de abstinencia a diversas drogas de abuso (Kranzler et al., 2014); como esquemas de reemplazo a opiáceos (ej: metadona); en la dependencia a la nicotina, la Agencia Nacional de Medicamentos, Alimentos y Tecnología Médica, ANMAT, de la Argentina, ha aprobado los reemplazos de nicotina por medio de parches, chicles, spray, caramelos, el bupropión y la vareniclina; para la dependencia al alcohol en la cual se encuentran aprobados por la ANMAT el disulfiram y la naltrexona, en el tratamiento de trastorno mental comórbido (patología dual) y en indicaciones complementarias donde interviene el psiquiatra como, por ejemplo, en ciertas deficiencias o carencias dietéticas (vitaminas B, D, etc.).

\section{Reunión clínica semanal}

La tarea del equipo terapéutico de un DAI de pacientes con TUS es un desafío en varios órdenes ya sea por algunas posibles características de los consultantes o por la manera en que éstos convocan a los profesionales intervinientes. Algunas de esas características son:
- Son pacientes graves de alto riesgo, en consumo, ambivalentes y con sentimientos encontrados respecto de iniciar tratamiento, con frecuencia con problemas legales, vinculares y laborales.

- En muchas ocasiones presentan comorbilidad psiquiátrica, como trastornos psicóticos, del estado del ánimo, de la personalidad, por déficit de atención con hiperactividad, etc.

- Muchos presentan problemas clínicos como consecuencia del consumo y otros tienen antecedentes clínicos de base que empeoran con el uso de sustancias. Algunos ejemplos son: enfermedades de transmisión sexual (ETS), hepatopatías, cardiopatías, neuropatías, etc.

- Algunos pacientes admitidos en el dispositivo manifiestan sentimientos de desconfianza, desgaste o temor; mostrándose a la defensiva ya que han transitado por experiencias terapéuticas previas difíciles y/o negativas, como internaciones en comunidades terapéuticas, desintoxicación aguda en clínicas psiquiátricas, consultas a profesionales de la salud mental que han generado desconfianza o han tenido una actitud "expulsiva" y/o punitiva vinculada al consumo.

- Con frecuencia los pacientes son traídos por terceros (familiares, amigos, conocidos) y, generalmente, tienen muy poca motivación de realizar tratamiento.

Estas características confrontan a los miembros del equipo de diversas maneras en su rol profesional:

- La formación en el tema específico de los TUS y su complejidad, generalmente abordada de manera insuficiente durante la carrera de especialista.

- Sus propios prejuicios y creencias como personas respecto a esta población de pacientes. 
- La necesidad de una alta tolerancia a la frustración, aunque si se tiene un genuino interés para encarar esta tarea compleja y ardua, también puede ser muy gratificante.

- La intención de generar un vínculo de confianza tal que permita realizar psicoeducación a aquellos pacientes traídos por terceros, quienes generalmente se encuentran en la etapa pre-contemplativa de Prochaska y motivarlos a realizar tratamiento, así como problematizar su consumo.

Teniendo en cuenta estas consideraciones se estableció una reunión semanal obligatoria para los profesionales: psicólogos, psiquiatras, coordinador general, director del dispositivo y otros profesionales como nutricionista, terapeuta familiar y toxicóloga. Previo al encuentro los equipos terapéuticos envían al coordinador las situaciones puntuales de alarma de cada paciente a su cargo. Este espacio ayuda a que todo el equipo del dispositivo intercambie ideas y todos los profesionales puedan aportar o sugerir intervenciones a tiempo, como el uso específico de herramientas o entrevistas adicionales que "re-encuadren" al paciente en el tratamiento.

Al finalizar la reuniones semanales del equipo asistencial en el DP, los profesionales responden dos preguntas: ¿Cuál es el paciente que más le preocupa? $\mathrm{Y}$ ¿Cuál de los profesionales del equipo requiere más apoyo? Ambas fortalecen la comunicación y la ayuda entre colegas.

\section{Ateneo clínico y cursos de formación}

El ateneo clínico mensual es una actividad exclusiva para los profesionales del DP. Esta actividad académica toma como referencia un caso clínico particular o un tema clínico relevante para revisar de manera bibliográfica. Se invita a referentes especialistas en psiquiatría o psicología a pensar el caso o tema en cuestión. Se desarrollan interrogantes y se intenta generar consenso entre todos para el abordaje integral de cada paciente.

En el DP se sostiene que es fundamental la exposición ante los colegas. En el ateneo clínico cada uno de los profesionales aporta desde su propio conocimiento, recorrido teórico y clínico. Permite las diferencias de conceptos y argumentos que ayuden a pensar desde diversas perspectivas.

Desde 2020 el dispositivo cuenta con una plataforma docente, en la cual se imparten cursos virtuales que apuntan a la formación continua de los profesionales que conforman el equipo.

\section{Conclusiones}

El DAI es una de las propuestas más acordes a la complejidad del tratamiento de patologías crónicas, especialmente porque pretende generar conciencia de enfermedad y cambios de hábitos de vida en el ámbito de la comunidad del paciente.

El DP se plantea para pacientes con características específicas. Los mismos no podrán iniciar tratamiento en el caso de: estar en riesgo cierto e inminente, cursando una descompensación de enfermedad clínica o psiquiátrica, si no cuentan con red de apoyo social o si no tienen la intención de iniciar un tratamiento que promueva la abstinencia del consumo de sustancias.

Las herramientas con las que cuenta el DP se basan en conceptos neurobiológicos actualizados, entrevista motivacional propuesta teóricamente por Prochaska y Di Clemente, las estrategias de "prevención de recaídas" de Marlatt y Gordon, el "manejo de contingencias" de Silverman, las aproximaciones motivacionales de Miller, la psicoeducación, el modelo de reducción de daños, el entrenamiento de modificación de sesgo cognitivo (CBM) y el modelo Patient Centered Care (PCC) el cual promueve el desarrollo de herramientas clínicas "centradas en el paciente".

El paciente en el DP es un agente activo del cambio, cuenta con psicoterapia individual, grupal, valoración psiquiátrica y toxicológica y talleres específicos para su tratamiento individual. Por lo tanto, se encuentra vinculado a un grupo de profesionales que permanentemente están en diálogo con él, en todas las etapas de su tratamiento.

En el marco de las teorías aplicadas para el desarrollo del dispositivo, se considera fundamental para obtener un mejor desempeño profesional clínico la investigación continua, las actividades formativas de los profesionales involucrados y las reuniones de equipo semanales.

El DAI del DP es una propuesta de tratamiento integral que el paciente realiza en su comunidad. Al ser condición de admisión contar con una red de apoyo se trabaja no solo con el paciente en particular sino con su entorno social, generalmente desgastado por tratamientos previos frustrados.

Conflictos de intereses: La/os autoras/es forman parte del equipo del Dispositivo Pavlovsky. 


\section{Referencias bibliográficas}

American Psychiatric Association. (1995). Practice guideline for the treatment of patients with substance use disorders: alcohol, cocaine, opioids.. The American journal of psychiatry, 152(11 Suppl), 1-59. https://doi. org/10.1176/ajp.152.11.1

Arias Gallegos, W. L.; Huamani Cahua, J. C., \& Choque Vera, R. (2018). Análisis psicométrico del test de Fagerström de dependencia a la nicotina en una muestra de estudiantes universitarios de Arequipa, Perú. Acta méd. Peru, 35(3), 174-179. http://www.scielo.org.pe/scielo.php?script=sci_arttext\&pid=S1728-59172018000300006\&lng=es\&nrm=iso $>$

Babor, T. F., Higgins-Biddle, J. C., Saunders, J. B., \& Monteiro, G. (2001). Cuestionario de identificación de los Trastornos debidos al consumo de alcohol. Pautas para su utilización en Atención Primaria. WHO/MSD/ MSB/01.6a. https://www.who.int/substance_abuse/activities/en/AUDITmanualSpanish.pdf

Bagnati, P. (2017). Evaluación neurocognitiva en adicciones. En: Adicciones, M. Waisman Campos, A. Benabarre Hernández (Edits.). Editorial Médica Panamericana.

Barnicot, K., \& Crawford, M. ( 2019). Dialectical behaviour therapy v. mentalisation-based therapy for borderline personality disorder. Psychol Med, 49(12), 2060-2068. doi: 10.1017/S0033291718002878.

Bratti-van der Werf, M. K., Laurens, M. C., Postel, M. G., Pieterse, M. E., Ben Allouch, S., Wiers, R. W., Bohlmeijer, E.T., \& Salemink, E. (2018). Augmenting Outpatient Alcohol Treatment as Usual With Online Alcohol Avoidance Training: Protocol for a Double-Blind Randomized Controlled Trial. JMIR Res Protocolos, 7(3), e55. https://www.researchprotocols. org/2018/3/e55?utm source $=$ TrendMD\&utm medium $=$ cpc\&utm campaign=JMIR TrendMD 0

Calvo-García, F., Costa-Ribot, J., \& Valero-Piquer, R. (2016). Motivos de abandono temprano de pacientes en tratamiento ambulatorio de adicción a sustancias. Revista Española de Drogodependencias, 41(1), 41-55. https:// roderic.uv.es/bitstream/handle/10550/54890/5577357.pdf?sequence=1

Campo-Arias, A., Barros-Bermúdez, J.A., \& Rueda-Jaimes, G. E. (2009). Propiedades psicométricas del cuestionario CAGE para consumo abusivo de alcohol: resultados de tres análisis Rev Colomb Psiquiatr, 38(2), 294-303.

Capece, J. (2008). Repensando las adicciones: el paradigma cognitivo y el trastorno por dependencia. Vertex Rev Arg Psiquiatr, 29(77), 527-536. http://www.polemos.com.ar/docs/vertex/vertex77.pdf

Capece, J., \& Pavlovsky, F. (2013). Farmacoterapia de los Trastornos por Dependencia de Sustancias. En: S. Wikinski y G. Jufe (Edit.). Tratamiento farmacológico de los Trastornos Psiquiátricos. (pp. 333-364). Edit Médica Panamericana.

Del Cueto, A. M., \& Fernández, A., M. (2002). Dispositivo grupal. Carrera de Psicología. Universidad. Nacional de Buenos Aires. http://www. terras.edu.ar/biblioteca/30/30DEL-CUETO-Ana-Maria-FERNANDEZ-Ana-Maria-El-dispositivo-grupal.pdf

Dieterich, M., Irving, C. B., Bergman, H., Khokhar, M. A., Park, B., \& Marshall, M. (2017). Intensive case management for severe mental illness. Cochrane Database of Systematic Reviews, Issue 1. Art. No.: CD007906. DOI: 10.1002/14651858.CD007906.pub3.

Echeburúa, E., De Corral, P., \& Amor, P. J. (2004). Prevención de recaídas en las ludopatías. Salud y drogas, 4(2), 97-116. https://www.redalyc.org/ pdf/839/83940206.pdf

Fishman, J., Reynolds, \& T., Redel, E. (1999). A retrospective investigation of an intensive outpatient substance abuse treatment program. American Journal of Drug and Alcohol Abuse, 25,185-196.

García Rodríguez, O. (2008). Tratamiento conductual de la adicción a la cocaína. Trastornos Adictivos, 10(4), 242-51. https://www.elsevier.es/es-revista-trastornos-adictivos-182-pdf-13131180

González, M., Martí-Esquitino, J., Botella, A., Infer, A., Couto, B., \& Pons, D. (2013). Modelo Matrix: Manual del terapeuta: sesiones educativas para familias 2013/03. https://www.researchgate.net/publication/314078984 Modelo_Matrix Manual del terapeuta sesiones_educativas para familias
Jasiner, G. (2008). Coordinando grupos. Una lógica para los pequeños grupos. Lugar Editorial.

Kaës, R. (1996) El grupo y el trabajo del preconsciente en un mundo en crisis. Revista de la Asociación Argentina de Psicología y Psicoterapia de Grupo. 19; 77-101.

Kranzler, H. R., Feinn, R., Gelernter, J., Pond, T., \& Covault, J. (2014). Topiramate's reduction of body mass index in heavy drinkers: Lack of moderation by a GRIK1 polymorphism. Experimental and Clinical Psychopharmacology, 22(5), 419-423. https://doi.org/10.1037/a0037309

López-Núñez, C., Martínez-Loredo, V., Weidberg, S., Pericot-Valverde, I., \& Secades-Villa, R. (2016). Voucher-based contingency management and in-treatment behaviors in smoking cessation treatment. International journal of clinical and health psychology : IJCHP, 16(1), 30-38. https://doi. org/10.1016/j.ijchp.2015.05.003, 16, 30-38.

Loureiro, C., García C., Adana, L., Yacelga, T., Rodríguez Lorenzana, A., \& Maruta, C. (2018). Uso del test de evaluación cognitiva de Montreal (MOCA) en América Latina: revisión sistemática. Rev Neurol, 66(12), 397 408. https://www.neurologia.com/articulo/2017508

Marchand, K., Beaumont, S., Westfall, J., MacDonald, S., Harrison, S., Marsh, D. C., \& Schechter, M. T., Oviedo-Joeke, E. (2019). Conceptualizing patient-centered care for substance use disorder treatment: findings from a systematic scoping review. Substance Abuse Treatment, Prevention, and Policy, 14(1), 37. DOI: 10.1186/s13011-019-0227-0. PMID: 31511016; PMCID:

Millar, W. R., Rollnick, S. (1999). La entrevista motivacional. Preparar para el cambio de conductas adictivas. Editorial Paidós.

NIDA. (2020). (s/d). Principios de tratamientos eficaces. En: Principios de tratamientos para la drogadicción: Una guía basada en las investigaciones https://www.drugabuse.gov/es/publicaciones/principios-de-tratamientos-para-la-drogadiccion-una-guia-basada-en-las-investigaciones/principios-de-tratamientos-eficaces. Último acceso: 15 de septiembre de 2021.

Nutt, D., King, L. A., Saulsbury, W., \& Blakemore, C. (2007). Development of a rational scale to assess the harm of drugs of potential misuse. The Lancet, 369(9566), 1047-1053. https://doi.org/10.1016/S0140-6736(07)60464-4

Organización Panamericana de la Salud (OPS). (s/d). Abuso de sustancias. https://www.paho.org/es/temas/abuso-sustancias. Último acceso: 13 de septiembre de 2021.

Pavlovsky, F. (2019). Disulfiram, historia, controversias y su utilización en la actualidad. Vertex Rev Arg Psiquiatr, 30(146), 275-289.

Pavlovsky, F. et al. (2020). El dispositivo Pavlovsky para el tratamiento de los consumos problemáticos, Manual de trabajo. Editorial Noveduc.

Portero Lazcano, G. (2015). DSM-5. Trastornos por consumo de sustancias. ¿Son problemáticos los nuevos cambios en el ámbito forense? Cuad med Forense, 21(3-4), 96-104. https://scielo.isciii.es/scielo.php?script=sci arttext\&pid=S1135-76062015000200002

Tarter, R.E., \& Hegedus, A. M. (1991). The Drug Use Screening Inventory. Alcohol Health and Research World, 15(1), 65.

Risso, M., Bulacia, A. V., Castelluccio, \& M. C., Valdez, P. (2019). Estudio comparativo de la continuidad de tratamiento entre pacientes con trastorno por consumo de sustancias y pacientes con patología dual del Hospital “J. A. Fernández", Argentina. Vertex Rev Arg Psiquiatr. 30(145), 165-171. http://www.polemos.com.ar/docs/vertex/vertex145.pdfhttp://www.polemos.com.ar/docs/vertex/vertex145.pdf

Sánchez, L., Díaz-Morán, S, Grau-López, L., Moreno, A., Eiroa-Orosa, F. J., Roncero, C., Gonzalvo, B., Colom, J., \& Casas, M. (2011). Tratamiento ambulatorio grupal para dependientes de cocaína combinando terapia cognitivo conductual y entrevista motivacional. Psicothema, 23(1), 107113 http://www.psicothema.com/

Stagnaro, J. C., Cia, A., Vázquez, N., Vommaro, H., Nemirovsky, M., Serfaty, E., Sustas, S. E., Medina Mora, M. E., Benjet, C., Aguilar-Gaxiola, \& S., Kessler, R. (2018). Estudio epidemiológico de salud mental en población general de la República Argentina. Vertex Rev Arg Psiquiatr, 24(142), 275299. http://www.polemos.com.ar/docs/vertex/vertex142.pdf 
Templos Nuñez, L., \& Marín Navarrete, R. (2014). Entrevista motivacional para la atención de las adicciones. En: A. Tena-Suck \& R. Marín-Navarrete (Edits.). Temas Selectos en Orientación Psicológica VIII: Orientación Psicológica y Adicciones (pp.13-18). Manual Moderno.

Timko C. (2008). Outcomes of AA for special populations. Recent developments in alcoholism : an official publication of the American Medical Society on Alcoholism, the Research Society on Alcoholism, and the National Council on Alcoholism, 18, 373-392. https://doi.org/10.1007/978-0387-77725-2 21

Urshel, H. C., Blair, J., \& McLellan, A.T., Trad: Morales JD. (2003). Indice de Severidad de adicción. 5ta Edición. Versión compatible al Censo Clínica/De Entrenamiento (Patrocinado por: Sistemas Quickstart, Inc.). https://studylib.es/doc/6371921/indice-de-severidad-de-adicci\%C3\%B3n-5ta-edici\%C3\%B3n-versi\%C3\%B3n-compa.
Veach, L. J., Remley, T. P., Jr, Kippers, S. M., \& Sorg, J. D. (2000). Retention predictors related to intensive outpatient programs for substance use disorders. The American journal of drug and alcohol abuse, 26(3), 417-428. https://doi.org/10.1081/ada-100100253.

Velázquez Benítez, D., Friman Rodríguez, N., \& González García, M. (2016). Programas de reducción de daños en las adicciones, un dilema ético. Correo Científico Médico de Holguín, 20(4), 804-809. 